\title{
Development of Underactuated Prosthetic Fingers with Joint Locking and Electromyographic Control
}

\author{
Bart Peerdeman ${ }^{1,2}$, Stefano Stramigioli ${ }^{1,2}$, Edsko E. G. Hekman ${ }^{1,2}$, Dannis M. Brouwer ${ }^{1,3}$ \& Sarthak Misra ${ }^{1,2}$ \\ ${ }^{1}$ University of Twente, Enschede, the Netherlands \\ ${ }^{2}$ MIRA-Institute for Biomedical Technology and Technical Medicine, the Netherlands \\ ${ }^{3}$ CTIT-Centre for Telematics and Information Technology, the Netherlands \\ Correspondence: Bart Peerdeman, Robotics and Mechatronics, University of Twente, PO Box 217, Enschede \\ 7500 AE, Overijssel, the Netherlands. Tel: 31-648-584-257. E-mail: b.peerdeman@utwente.nl
}

Received: January 21, 2013

Accepted: February 6, 2013 Online Published: April 25, 2013

doi:10.5539/mer.v3n1p130

URL: http://dx.doi.org/10.5539/mer.v3n1p130

\begin{abstract}
Modern hand prostheses possess a large number of degrees of freedom. These degrees of freedom cannot simply be actuated by a single motor each, since their combined size and weight would exceed the limitations of an anthropomorphic prosthesis. Some hand prostheses try to remedy this by way of underactuation of the fingers or addition of entirely passive fingers, but this reduces the hand's ability to execute different grasp types.

A joint locking system is presented, allowing certain degrees of freedom to be fixed during actuation of an underactuated finger. These locks are actuated by miniature solenoids, and allow the fingers to support a variety of grasp types. In this paper, these locks are implemented in a two-fingered prosthesis prototype, which is able to perform several grasping motions. This prototype is controlled by pre-recorded electromyographic signals, which control the grasp type and opening/closing of the hand.

Various grasping experiments show that the prototype is able to execute three essential grasp types for daily living with a single main actuator, and can be intuitively controlled by means of six different electromyographic signals. This prototype demonstrates new joint locking mechanisms and control systems that can provide an anthropomorphic, myoelectric hand prosthesis with minimal actuation and intuitive control.
\end{abstract}

Keywords: hand prosthesis, underactuation, grasping, joint locking, electromyography

\section{Introduction}

Modern hand prostheses are becoming more and more anthropomorphic in their design (RSLSteeper, 2012; Touch Bionics Inc., 2011; Otto Bock, 2011). The most daunting aspect of this trend is the increase in degrees of freedom (DOFs) that have to be actuated and controlled. DC motors are the most common type of prosthesis actuator, and only a few of these large and heavy actuators can be placed inside the palm of the hand.

This can be remedied by actuating multiple degrees of freedom with a single actuator, a technique known as underactuation. This technique has been implemented in other prosthesis projects with varying results. A linkage, such as that implemented in the Southampton hand (Light \& Chappell, 2000), couples the finger joints' motion using rigid bars. This allows the more distal joints of the finger to flex when the proximal joints' movement is obstructed. To couple multiple finger linkages, a whippletree mechanism can be implemented, which distributes the actuator force appropriately. The movement pattern of the fingers can be influenced by changing the structure of the linkage.

Underactuation can also be achieved via a series of freely rotating pulleys on each joint, which are connected by way of a single communal tendon (Carrozza et al., 2006). Since this system can only apply forces along the flexion direction due to the nature of the tendon, a second tendon or extension springs are required to allow full motion of the finger. The finger's flexion motion depends on the friction and external forces on the joints, but can also be influenced by the relative radii of the pulleys.

An alternative to the underactuation of multiple joints is the inclusion of passive or coupled joints (Pons et al., 2004). The distal interphalangeal joints are often implemented as such, because the angle between the distal and medial phalanges is also strongly coupled in the human hand. Similarly, the movement of the little and ring 
fingers can be coupled while still resembling the human hand's freedom of motion.

Another advantage of some of these systems is that the finger will flex in such a way as to accommodate the shape of a grasped object. However, the main drawback of these underactuated systems is the loss of control over the fingers' motion; in free space, such underactuated fingers will follow a set trajectory determined by their mechanical structure. In modern commercial prostheses with underactuated fingers (RSLSteeper, 2012; Touch Bionics Inc., 2011; Otto Bock, 2011) this trajectory is designed to be usable for both power and precision grasping, but is less then ideal for either.

The ability to selectively disable the motion of certain joints allows a hand to match the fingers' trajectory to the grasp type selected by the user, as seen in Figure 1. Also, the motion of certain fingers can be completely disabled, which allows all fingers to be connected to a single main actuator. There has been some research on mechanisms that passively disengage joints (Ulrich et al., 1989; Chu et al., 2008), as well as active electrostatic joint locking on robotic grippers (Aukes et al., 2012). In (Peerdeman et al., 2012b), the use of miniature mechanical joint locks is explored for use in hand prostheses. The limitations imposed by an anthropomorphic hand prosthesis lead to a lock actuation system integrated into the phalanges of the fingers, as well as a lock design which eliminates the need for continuous actuation.

These joint locks have been implemented in human-sized fingers, based on a friction amplification principle (see Figure 2). The locks are actuated by solenoids integrated into the body of each phalanx. Though these solenoids provide only a minimal stroke and force, these are sufficient due to the self-locking properties of the friction lock.

When making a prosthesis with a large number of available DOFs, it is important to consider their controllability by the user as well. Detection of myoelectric (ME) signals is the most common method of control for modern externally actuated hand prostheses (Peerdeman et al., 2011) It is a non-invasive method of obtaining signals directly correlated to muscle activity, though current classification methods only allow a few usable control signals to be distinguished. This control bottleneck strongly limits the variety of prosthesis motions that can be achieved. To compensate for this, a control system can be constructed around a discrete selection of grasp types, which can be selected and activated manually; the execution of these grasps will be done automatically.

In this article, the mechanical design of a new two-fingered prosthesis prototype is described, using tendon-pulley underactuation and joint locking. An ME control system is implemented as well, using electromyographic (EMG) signals to control various grasp types and motions of the prototype. In Section 2, the structure of the prototype is described, from the design of the phalanges to the joint locking and actuation systems. Section 3 describes the implementation of the control system, consisting of an EMG classifier, high-level grasp planner, and low-level finger controller. In Section 4, the experimental setup and test protocol are described. The results are shown in Section 5. A discussion of the results is found in Section 6, as well as the conclusion and directions for future work.
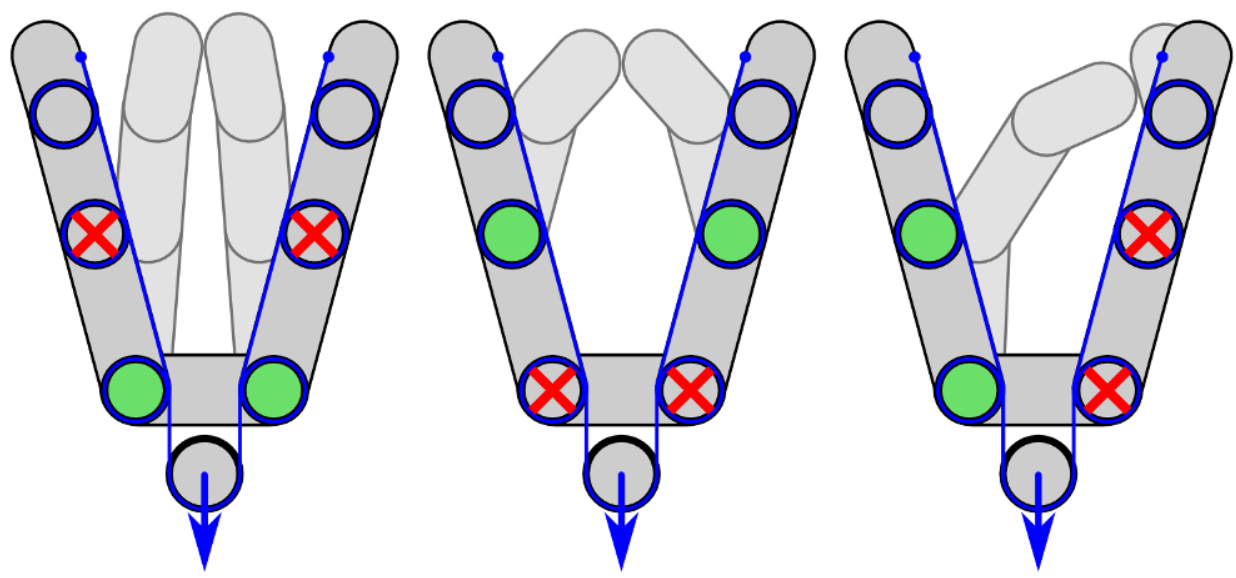

Figure 1. Various finger trajectories that can be attained in a two-fingered, underactuated hand by means of joint locking. The paths of the actuator tendons are shown as blue lines. Red crosses indicate locked joints, and green circles indicate unlocked joints 


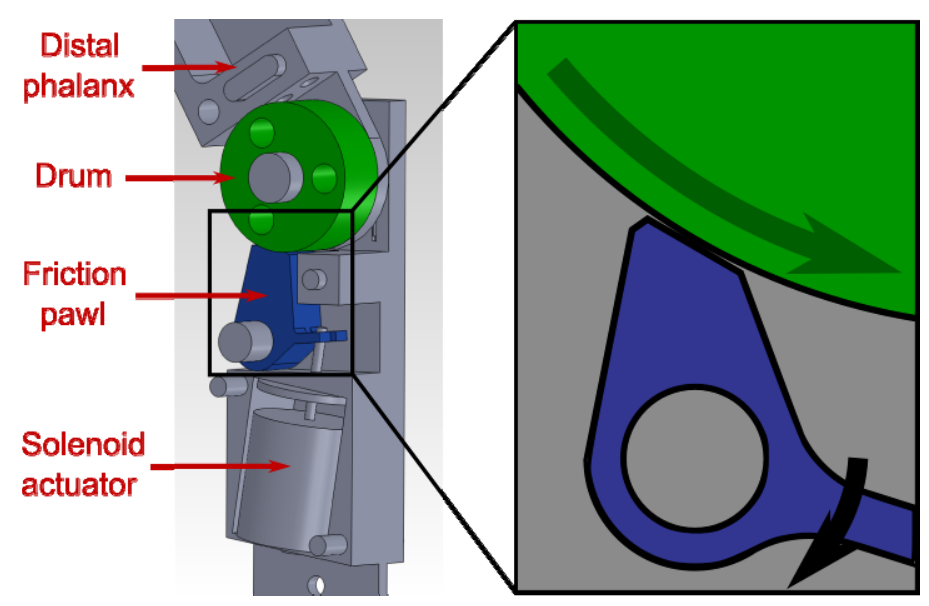

Figure 2. Friction amplification joint locking concept: If the friction pawl is brought into contact with the drum by the solenoid actuator, friction between pawl and drum will pull the two together, locking the joint

\section{Mechanical Design}

The design of the prosthesis prototype is based on achieving a large variety of finger motions with minimal actuation. The prototype consists of two fingers with two flexion/extension DOFs each, which are connected by a single tendon to the main actuator; a diagram of the prototype is shown in Figure 3. The underactuated nature of this tendon-pulley system precludes individual control of the fingers' DOFs. This is compensated for by including joint locking mechanisms to block the flexion motion of specific joints, while leaving the others free to move. By locking different combinations of joints, the fingers can be made to perform a variety of flexion trajectories relevant to grasps in activities of daily living (Peerdeman et al., 2011).

\subsection{Finger Structure}

The external dimensions of the finger need to be close to those of the human finger, for reasons of usability and cosmetic appearance. This puts tight constraints on the size of the joint locking mechanism and its solenoid actuators. Therefore, a finger joint diameter of $15 \mathrm{~mm}$ is chosen. The phalanx dimensions can be found in Table 1 , and for reference, phalanx dimensions for a male index finger are shown in Table 2. The internal structure of one of the fingers' medial and proximal phalanges is shown in Figure 4. Each actuated joint contains a freely rotating pulley, a drum for the joint locking mechanism, and a rotational spring to provide an extension force. The phalanges are constructed out of aluminum, whereas the joint shafts and lock components are made of (hardened) steel.

Table 1. Dimensions of the prototype's phalanges

\begin{tabular}{llll}
\hline & Length $(\mathrm{mm})$ & Width $(\mathrm{mm})$ & Height $(\mathrm{mm})$ \\
\hline Distal & 28.0 & 15.0 & 15.0 \\
Medial & 33.0 & 18.0 & 17.0 \\
Proximal & 50.0 & 18.0 & 15.0 \\
Metacarpal & 42.5 & 18.0 & 15.0 \\
\hline
\end{tabular}

Table 2. Average dimensions of male index phalanges, with a hand length of $195 \mathrm{~mm}$ and breadth of $88.5 \mathrm{~mm}$ (Buchholz \& Armstrong, 1991)

\begin{tabular}{llll}
\hline & Length $(\mathrm{mm})$ & Width $(\mathrm{mm})$ & Height $(\mathrm{mm})$ \\
\hline Distal & 18.9 & 16.4 & 13.7 \\
Medial & 27.9 & 18.2 & 16.3 \\
Proximal & 47.8 & 18.5 & 18.7 \\
\hline
\end{tabular}




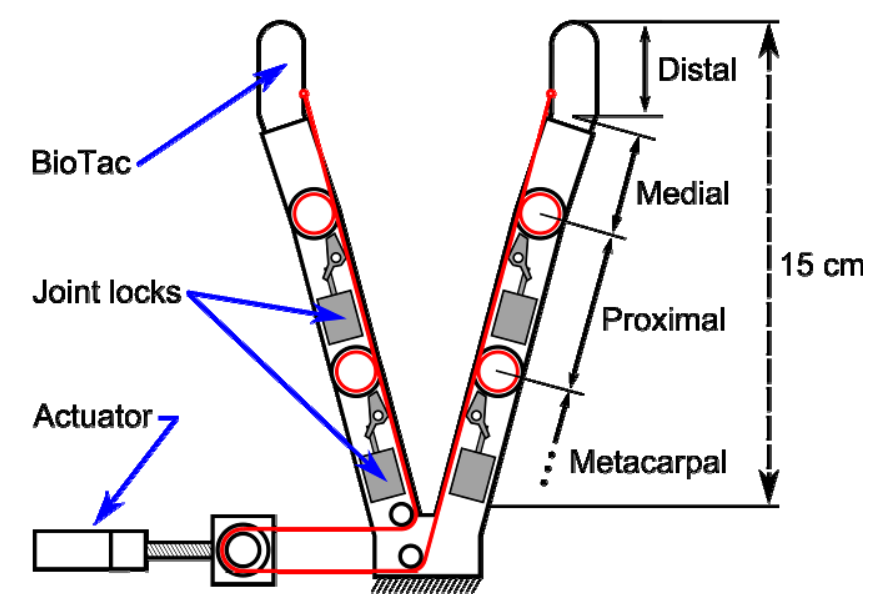

Figure 3. The two-fingered prototype, showing internal mechanisms and tendon routings. The phalanges are indicated on the right, while their dimensions are listed in Table 1

\subsection{Joint Locking}

In (Peerdeman et al., 2012b) two joint locking concepts were evaluated; a gear-based system and a friction-based system. The gear-based system has an inherent indexing resolution and was found to require a higher actuation force. Therefore, the friction-based mechanism has been chosen for the prototype. The principle of friction locking is illustrated in Figure 2. This mechanism consists of two main components: a drum fixed to the distal phalanx of a joint, and a friction pawl connected to the proximal phalanx. The friction pawl can be rotated around its pivot by the solenoid actuator. When the friction pawl is brought into contact with the drum, the rotation of the drum will pull the pawl closer to it, increasing the friction force between both until the joint's motion is completely blocked. This self-locking property means actuation is only needed to bring the lock parts into contact, which reduces the solenoids' actuation requirements. Once the actuation force is removed from the joint, an extension spring will separate the drum and pawl, releasing the lock.

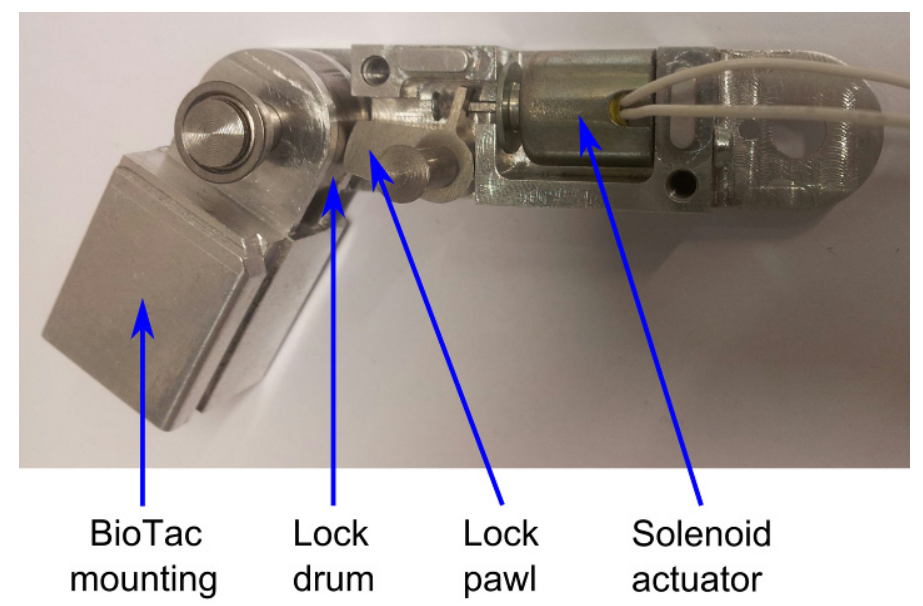

Figure 4. Internal structure of one of the fingers' medial and proximal phalanges, showing the friction lock components

Based on initial test results, a new set of friction pawls has been designed for implementation in a phalanx-sized enclosure. The contact surface of these pawls follows a logarithmic spiral around its rotation axis (Peerdeman et al., 2012b), as shown in Figure 5. The effect of this surface is shown in Figure 5 as well: the effects of changes in the distance between drum and pawl (due to play and tolerances in the components, for example) on the contact angle are minimized. 


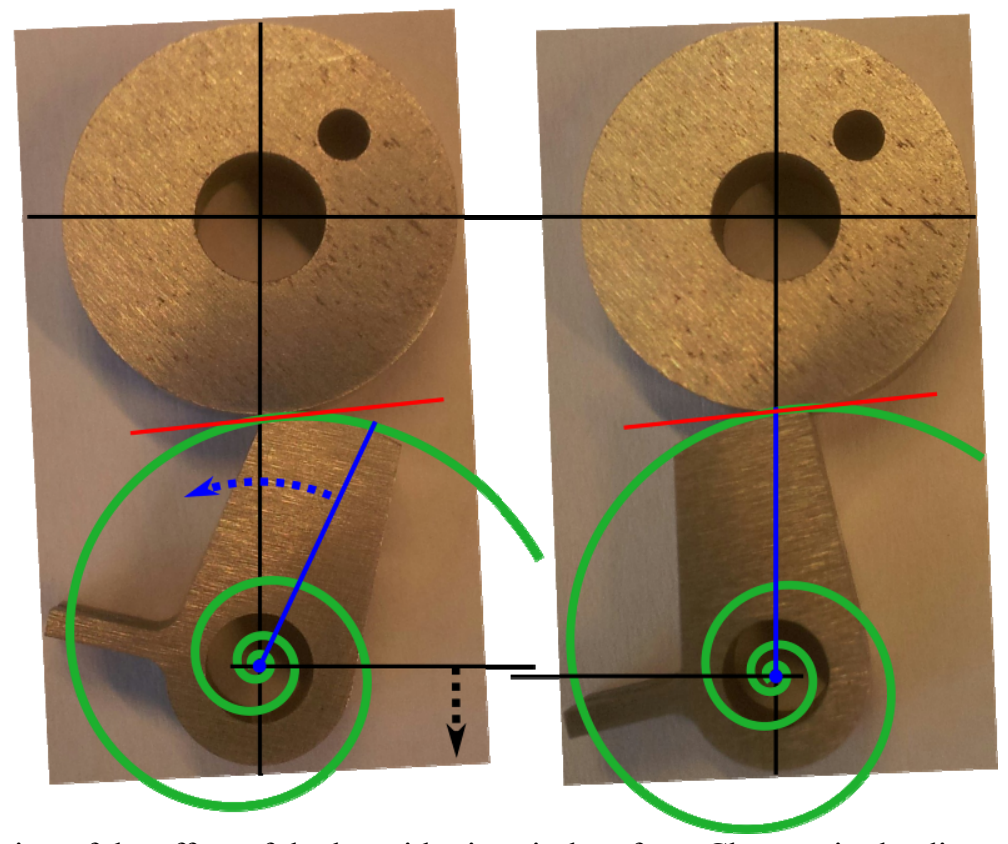

Figure 5. Demonstration of the effect of the logarithmic spiral surface: Changes in the distance between the rotation axes of the drum and the pawl (black arrow) are compensated by rotation of the pawl (blue arrow), without affecting the contact angle (red line). The green line illustrates the spiral curve

\subsection{Actuation}

The two fingers of the prototype are actuated by a single DC motor. The motor is a Maxon EC-max 22 (Maxon Motor AG, Sachseln, Switzerland) connected to a spindle drive for translational motion. This motor moves a pulley block, which actuates the single flexion tendon routed around all the joint pulleys, as seen in Figure 3. The joint locks can then be used in order to actuate only a single finger or enable different grasp types. The locks themselves are each actuated by an in-phalanx pull-type solenoid with extension spring.

\subsection{Sensors}

Effective control of the fingers requires that force and position information be made available to the control system. To this end, each joint contains a Hall-effect based angle sensor, which can be used to determine the pose of the finger. The medial phalanx of each finger is an adapter for a BioTac tactile sensor system (SynTouch LLC, Los Angeles, CA, USA) (Wettels, 2011). The BioTac sensor replaces the distal phalanx of the finger. The device features two sensing modalities relevant to prosthesis control: a series of 19 electrodes that collectively determine the magnitude and location of external pressures on the fingertip, and a main pressure sensor that detects high-frequency variations in the applied pressure. With this information, it is possible to automatically control the fingers' interaction with the environment. However, the user should also be able to control the automated motion of the fingers. Therefore, a two-level control system has been implemented, which is discussed in the next section.

\section{Control}

The prototype's control system is based on a previously developed controller, which was used on the University of Bologna (UB) Hand IV in (Peerdeman et al., 2012a). The prototype's range of motions is divided into distinct grasp types, which can be used to grasp a variety of objects, as seen in Figure 6. The following grasp types are derived from a general set of activities of daily living (Peerdeman et al., 2011):

- Cylinder grasp: For this power grasp the thumb is opposed to the other fingers, and the object to be grasped is surrounded by the hand.

- Lateral grasp: In this grasp, the fingers are fully flexed, and the unopposed thumb is used to hold flat objects such as keys or cards.

- Tripod grasp: This precision grasp uses the index and middle fingers opposed to the thumb. The fingers' distal and medial joints remain extended, while the proximal joints are flexed.

However, each of these grasp types requires different joint locking configurations and finger trajectories, which 
can be demonstrated on the prototype as follows:

- Cylinder grasp: No joints are locked; the fingers show normal underactuated behavior. This allows the grasping ability of the finger to be evaluated.

- Lateral grasp: In this grasp, it is important to exclude certain fingers from the grasp motion at different times. One finger will be completely locked, while the other finger remains free to move. This configuration shows that multiple fingers can be individually actuated by a single motor.

- Tripod grasp: This grasp requires the actuation of only the proximal joints; the distal joints will be locked. This demonstrates the ability to increase the controllability of underactuated fingers by temporarily reducing their DOFs.

For the remainder of the paper, the grasp types will be used to refer to these locking configurations.

EMG signals from the user are used to select one of these grasp types, and to determine when to open and close the selected grasp. Once a grasp has been selected, the high-level controller plans the grasp, actuating the joint locks based on the motion of each of the fingers. Then, the low-level controller uses the DC motor to control the fingers to the desired end positions with the appropriate forces.

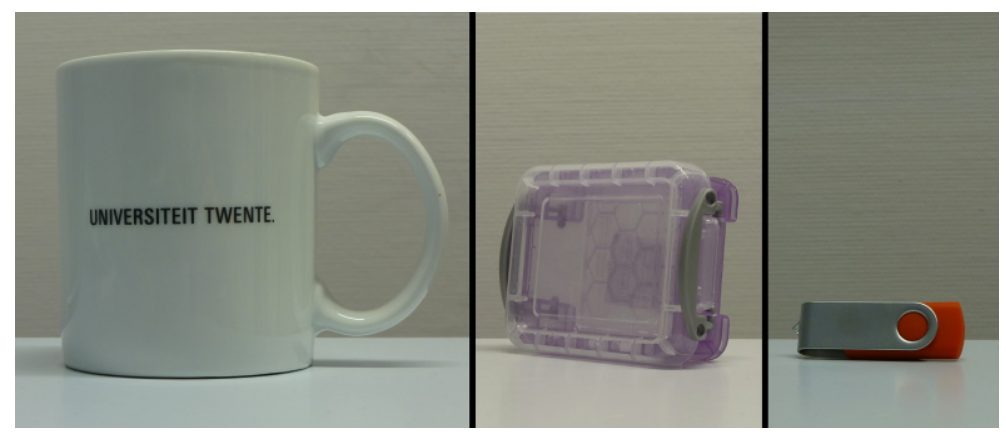

Figure 6. Three objects used in the grasping experiments. From left to right: mug ( $82 \mathrm{~mm}$ diameter), box ( $30 \mathrm{~mm}$ thickness), and USB flash drive (8 $\mathrm{mm}$ thickness)

\subsection{Electromyographic Classification}

EMG classification is used to couple the activation of different muscle groups in the forearm to the input signals of the high-level control system. In (Boere et al., 2010) multi-channel EMG was used to classify up to eight different muscle contraction types by means of an electrode array placed on the forearm. Based on this system, an EMG classifier has been developed using pre-recorded test data. In order to control the prototype, six different signals need to be identified:

- Cylinder: This signal selects a grasp without any joint locking.

- Lateral: With this signal, all joints of one finger are locked.

- Tripod: This signal locks the proximal joints of each finger.

- Close: To flex the unlocked joints of each finger.

- Open: To extend all finger joints.

- Stop: To abort the grasp and return the hand to its fully extended position.

Each of these signals corresponds to a certain muscle contraction type. A test set is constructed from a series of known pre-recorded signals. This test set is used to train a linear discriminant analysis classifier (Boere et al., 2010). This classifier can then be used to identify the signal type of a new contraction signal.

\subsection{High-Level Control}

Considering the limits of EMG classification, the high-level control system should be operable with only a few input signals. This can be accomplished by combining user-controlled and automated actions to perform a grasp. The grasp type is actively selected by the user, but execution of the grasp is performed automatically once a closing signal is received; this keeps grasp control intuitive to the user. The possible actions of the prototype are organized in a state machine, which can be seen in Figure 7. The states are described as follows: 
- Preshaping: Once a grasp type signal (Cylinder, Lateral or Tripod) is received, the system moves to this state immediately. The joint locks are automatically moved into the right positions for the desired grasp. Once the locks are in position, the system moves to the Ready state.

- Ready: In this state, the hand remains in the preshaping position. From here, a new grasp type can also be selected. Once a Close signal is received, the system moves to the Closing state.

- Closing: The grasp is automatically closed. If the user wishes to stop the grasp, an Open signal will move the system to the Opening state to extend the fingers. Once each finger involved in the grasp reports sufficient external force to establish contact, the system moves on to the Hold state.

- Hold: The fingers will automatically exert a constant force on the held object. If the user gives an Open signal, the system moves to the Opening state and the object is released.

- Opening: This state ends the grasp by automatically extending the involved fingers. The system moves to the Ready state once the fingers are fully extended.

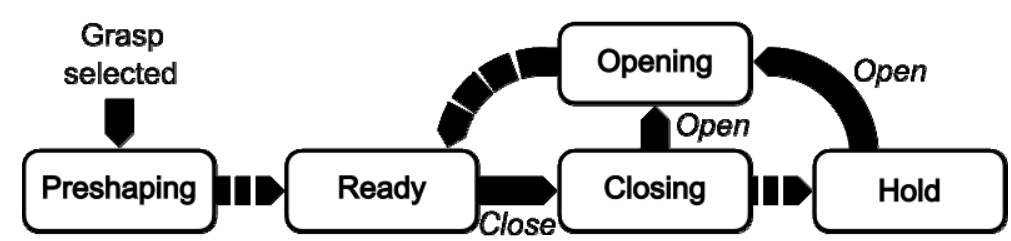

Figure 7. Grasp control state machine: Dashed arrows are automatic state changes, while the Open and Close signals originate from the electromyographic classifier

\subsection{Low-Level Control}

The low-level controller is tasked with moving the fingers to their desired positions, while applying the proper forces to any external obstructions. Initially, a force controller was implemented, which moves the fingers at a fixed velocity while no external forces are present. Once contact is established, the fingertip force is controlled to a set value.

However, impedance control is a more appropriate way of approaching interaction with the environment (Hogan, 1985), and therefore such a controller has been implemented on the prototype instead. The controller derives the force to be applied to the fingers $(\boldsymbol{F})$ from the difference between their desired state $\left(\boldsymbol{x}_{\boldsymbol{d}}, \boldsymbol{x}_{\boldsymbol{d}}{ }_{\boldsymbol{d}}\right)$ and measured state $\left(\boldsymbol{x}, \boldsymbol{x}^{\prime}\right)$ as follows (Peerdeman et al., 2012a):

$$
F=D\left(x_{d^{\prime}}-x^{\prime}\right)+K\left(x_{d}-x\right)
$$

If EMG amplitude information is available, the user can control the applied force directly by changing the value of the controller's stiffness $(\boldsymbol{K})$ or damping $(\boldsymbol{D})$ constants.

\section{Experiments}

This section describes the experimental setup, as well as the experiments used to evaluate the prototype's mechanical design and control system.

\subsection{Experimental Setup}

The experimental setup consists of the two-fingered prototype, its actuation systems (DC motor and solenoids), and its sensing systems (Hall sensors, BioTacs). These are connected to a National Instruments ELVIS DAQ device (National Instruments Corporation, Austin, TX, USA), which is controlled via Matlab (The MathWorks, Inc., Natick, MA, USA). An image of the two-fingered prototype indicating relevant subsystems can be seen in Figure 8. 


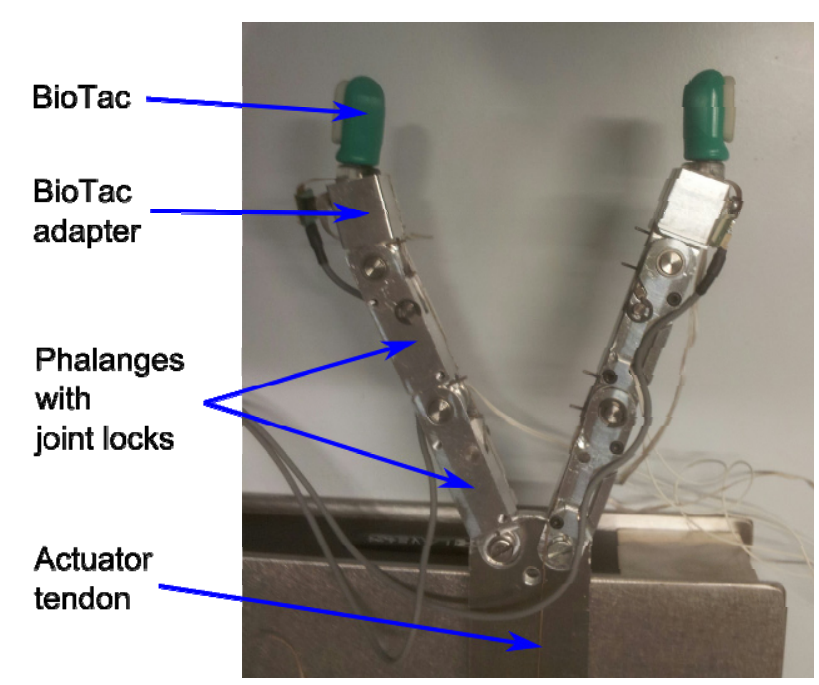

Figure 8. Two-fingered prototype experimental setup: Actuation is provided externally, by a DC motor and spindle drive connected to the actuator tendon

\subsection{Test Protocol}

\section{Control system}

The performance of the EMG classifier and the high-level state machine controller are evaluated by supplying pre-recorded EMG signals to achieve a sequence of grasping motions. These sequences are geared towards basic activities of daily living (Peerdeman et al., 2011). The EMG classifier's accuracy and response time are significant parameters, and are tested by repeatedly classifying a random sequence of pre-recorded EMG signals. In earlier research on similar classification by Boere et al. (2010), the accuracy was found to exceed $90 \%$. The high-level state machine is evaluated by inspection, and the execution time of the entire control system is measured as well.

Joint locking

The properties of the joint locking system are evaluated by locking different combinations of joints, and observing the resulting finger trajectories without obstructions. Repeated execution of these motions is used to investigate the effectiveness of the locks, as well as the consistency of the trajectories. Important considerations are the compliance of locked joints, which can be caused by play in the locking mechanism (Peerdeman et al., $2012 b$ ), and the locks' behavior at high joint torques.

\section{Grasping}

The pose of the prototype's fingers and the forces exerted on them are evaluated during grasping. The joint locks and DC motor are controlled to accomplish different grasp types. Although the grasp types used here are a simplification of those used with an anthropomorphic hand prosthesis, the basic variations that are tested here can be combined to allow a single main actuator to perform cylinder, tripod, and lateral grasps with five fingers as well.

An object is placed in between the fingers, and the joint angles and fingertip forces are measured using the Hall sensors and BioTacs, respectively. A specific object is chosen to illustrate the applications of each of the three grasp types: a mug for the cylinder grasp, a small box for the tripod grasp, and a USB flash drive for the lateral grasp. These objects are shown in Figure 6.

\section{Results}

This section summarizes the results of the experiments mentioned in Section 4, evaluating the control system, joint locking mechanism, and grasp performance of the prototype.

\subsection{Control System}

In these experiments, the accuracy and processing time of the EMG classifier are evaluated. A test set is created from a $90 \mathrm{~s}$ training recording divided into $50 \mathrm{~ms}$ windows, with the associated classes known in advance. This test set is used to train the classifier; afterwards, another window can be loaded, and the appropriate class is 
determined.

The performance of EMG classification is tested by entering a set of 25 random EMG windows into a trained linear discriminant analysis classifier 100 times. The results of one of these tests can be seen in Figure 9. The average accuracy of the classifier was found to be $92.53 \%$, with the average classification time for a single set being $1.6 \mathrm{~ms}$. This computation time is negligible compared to the window size required for classification. The only significant time delay in the high-level control state machine was found between moving the state machine to the Closing or Opening states and the start of motor activation, which takes approximately $96 \mathrm{~ms}$.

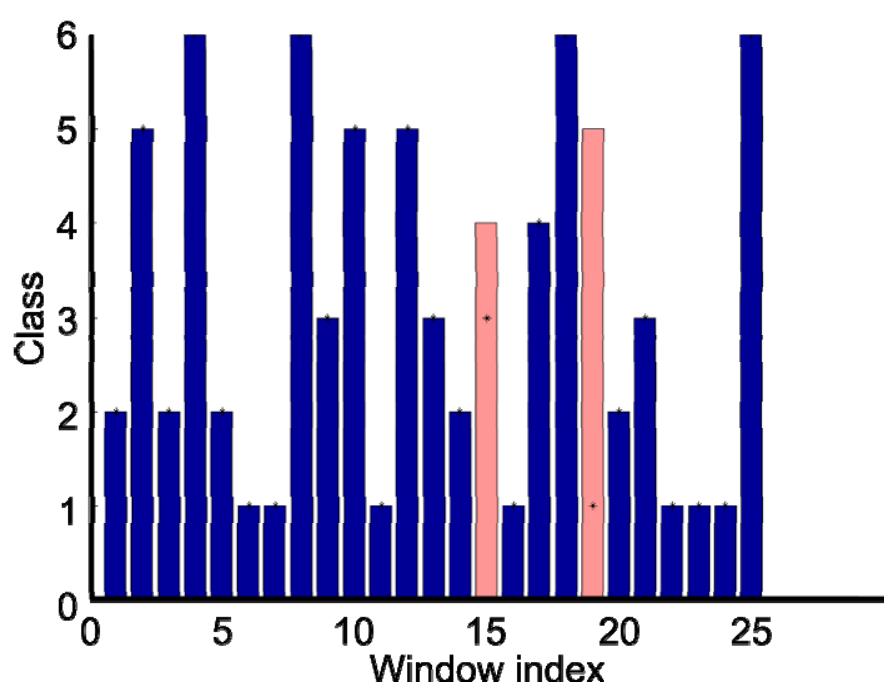

Figure 9. Control system experiment: A test of the linear discriminant analysis classifier with a set of 25 random electromyographic signal windows (Boere et al., 2010). Blue bars denote correct classifications; red bars denote errors

\subsection{Joint Locking}

To evaluate the functionality of the joint locks, the cylinder, tripod, and lateral grasps are performed without an object to grasp. The fingers are controlled to stop as soon as contact between the fingers is detected. The results of these tests can be seen in Figure 10. In the cylinder and lateral grasps, the joint angles of the freely moving fingers tend to stay equal. In the case of the tripod grasp, the joint locking ensures the proximal joint moves further than the locked distal joint. In both the lateral and the tripod grasp movement of the locked joints is still noticeable, though limited to 10 and 15 degrees, respectively. In general, even without object interaction, the functional differences between the three grasp types can be observed. Therefore, the joint locks can be considered an effective method of improving the range of motion of underactuated fingers.
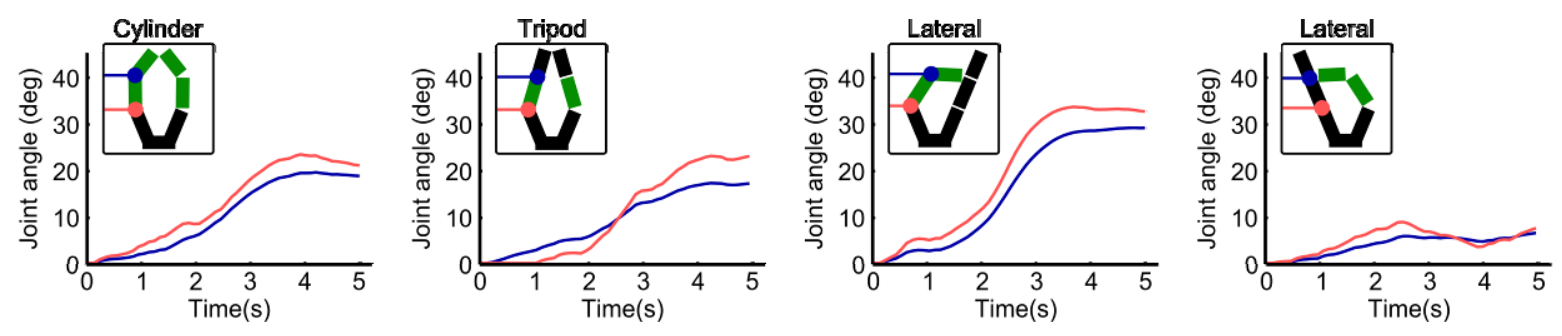

Figure 10. Joint locking experiment: The cylinder, tripod, and lateral grasps performed without grasping an object. The graphs indicate the fingers' joint angles: the blue line represents the distal finger joint, and the red line represents the proximal finger joint. For the lateral grasp, the locked finger's joint angles are also shown in the far right graph. The pictographs indicate the grasp type: green phalanges are in motion, and black phalanges are fixed 


\subsection{Grasping}

In these experiments, the grasping performance of the prototype is evaluated. The three grasp types are performed on various objects seen in Figure 6.

\subsubsection{Grasping Experiment 1 (Grasping the Mug Object)}

The results of this experiment can be seen in Figure 11. Because of the mug's size, the joints do not move more than 15 degrees. In the cylinder grasp both joints move roughly equally in the unlocked situation, and a stable grasp is established within $1 \mathrm{~s}$ after contact. For the tripod grasp the distal joints are locked, though some joint motion can still be observed. This is due to the joint compliance mentioned in Section 2.2. However, in this case the locking of the distal joints has little effect on the performance of this grasp. The left finger is locked entirely for the lateral grasp. The unlocked finger pushes the mug towards the base of the locked finger, and the resulting grasp is unfit for picking up the mug.
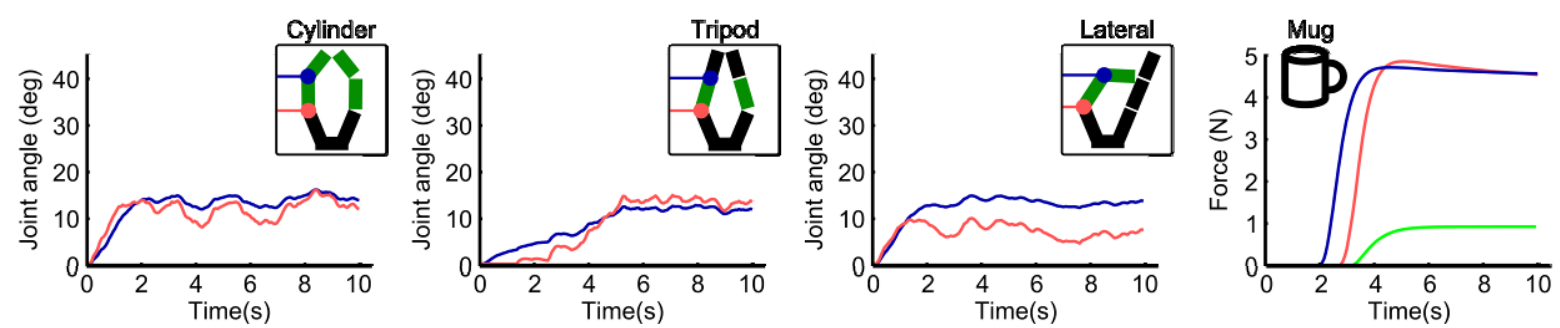

Figure 11. Grasping experiment 1: The cylinder, tripod, and lateral grasps performed on the mug object, showing joint angles and fingertip forces. The lateral grasp's locked finger is omitted, as its joint angles are the same as in Figure 10. In the joint angle graphs, the blue line represents the distal finger joint, and the red line represents the proximal finger joint. For the fingertip forces, the blue line represents the cylinder grasp, the red line represents the tripod grasp, and the green line represents the lateral grasp. The pictographs indicate the grasp type: green phalanges are in motion, and black phalanges are fixed

\subsubsection{Grasping Experiment 2(Grasping the Box Object)}

The results of this experiment can be seen in Figure 12. During cylinder grasping both joints initially move together, as with grasping the mug, but as soon as contact is established the proximal joints are extended in favor of continued distal joint flexion. This is a consequence of the fingers' underactuation and will destabilize the grasp, as only the tips of the fingers stay in contact with the object. With the tripod grasp, the locked joints prevent the proximal joints from being extended, which results in a stable grasp requiring less force. The proximal joint rotation is also higher than in the cylinder grasp, and does not reverse after contact. However, the residual compliance of the joint locks still allows the distal joints to flex up to approximately 20 degrees. During the lateral grasp the active finger shows similar behavior to the cylinder grasp, but the grasp requires less force because of increased fingertip contact with the object. The locked finger still has some compliance, but does not contribute to the grasp.
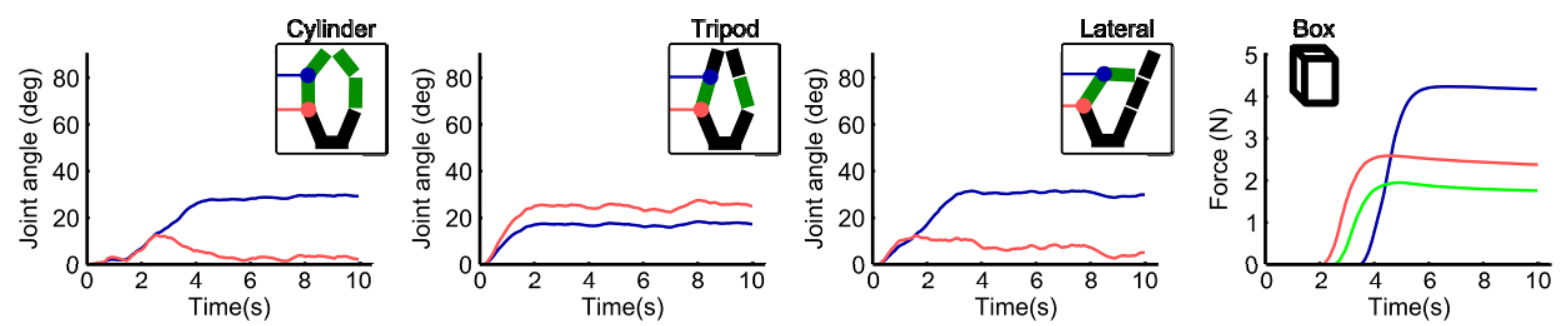

Figure 12. Grasping experiment 2: The cylinder, tripod, and lateral grasps performed on the box object, showing joint angles and fingertip forces. The lateral grasp's locked finger is omitted, as its joint angles are the same as in Figure 10. In the joint angle graphs, the blue line represents the distal finger joint, and the red line represents the proximal finger joint. For the fingertip forces, the blue line represents the cylinder grasp, the red line represents the tripod grasp, and the green line represents the lateral grasp. The pictographs indicate the grasp type: green

phalanges are in motion, and black phalanges are fixed 
The difference between the cylinder and tripod grasp types is shown in more detail in Figure 13. First, a cylinder grasp is performed on the box object. Because none of the joints are locked, the finger's underactuation causes it to continue flexing after contacting the object, which leads to an unstable grasp. Afterwards, the distal joint of each finger is locked to achieve a tripod grasp; this results in a firm hold on the object.
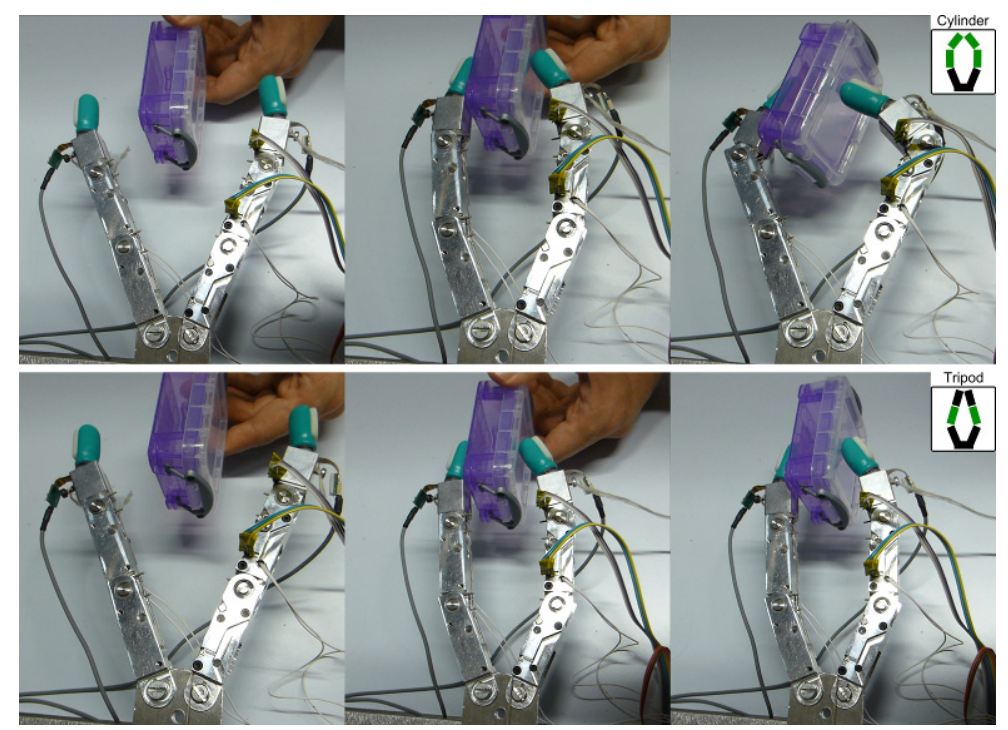

Figure 13. Video frames of the box object being grasped with a cylindrical grasp (top) and a tripod grasp (bottom). For each grasp, the image frames are spaced 1 second apart. The pictographs indicate the grasp type: green phalanges are in motion, and black phalanges are fixed

\subsubsection{Grasping Experiment 3 (Grasping the USB Drive Object)}

The results of this experiment can be seen in Figure 14. With the cylinder grasp, the smaller size of the object increases the problems experienced during grasping of the box, which results in repeatedly dropping the USB drive. The cylinder grasp is therefore unfit for this object. For the tripod and lateral grasps, the only difference is additional joint flexion due to the object's smaller size; the grasp stability and fingertip forces are unaffected.
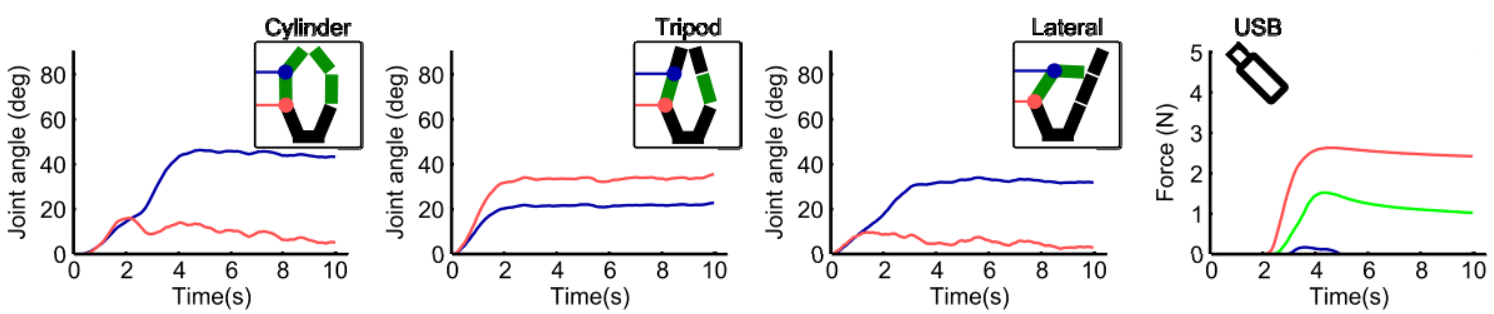

Figure 14. Grasping experiment 3: The cylinder, tripod, and lateral grasps performed on the USB drive object, showing joint angles and fingertip forces. The lateral grasp's locked finger is omitted, as its joint angles are the same as in Figure 10. In the joint angle graphs, the blue line represents the distal finger joint, and the red line represents the proximal finger joint. For the fingertip forces, the blue line represents the cylinder grasp, the red line represents the tripod grasp, and the green line represents the lateral grasp. The pictographs indicate the grasp type: green phalanges are in motion, and black phalanges are fixed

\section{Discussion}

In this section, the results are used to evaluate the general grasp performance of the prototype, as well as its individual subsystems. 


\subsection{Experiments}

\subsubsection{Control System}

The EMG classifier showed an accuracy of over $90 \%$, though it could still lead to occasional missed or misinterpreted commands. This can be addressed by evaluating several $50 \mathrm{~ms}$ signal windows for a single command, increasing the reliability of the classifier. The time required to evaluate a signal window was shown to be negligible relative to the length of the windows themselves. The high-level controller was functional in allowing the user to engage and release the chosen grasp, though qualitative evaluation of the system would require additional test subjects. The time delay between switching to the Closing or Opening state and actual motor activation can reduce the intuitiveness of the system and should be minimized. The low-level controller was able to reliably stabilize the applied grasping force based on the BioTac's sensor data, irrespective of the orientation of the fingertips with regard to the object.

\subsubsection{Joint Locking}

The results show the effectiveness of the joint locks in establishing finger motions relevant to the different grasp types. Also, they prevent motion of the fingers after establishing contact due to their underactuation. The different objects used in grasp testing also illustrate the use of different grasp types for activities of daily living. The joint locks did show between 10 to 15 degrees of compliance before joint motion stopped, though this did not prevent any of the grasp types from being performed.

\subsubsection{Grasping}

Stable grasps were established for 7 out of 9 combinations of grasp types and objects. A notable exception to this is the cylinder grasping of the box object, because the fingers' underactuation prevented the fingertips from establishing proper contact with the box. The forces exerted on the objects varied between $5 \mathrm{~N}$ for the mug, and $2 \mathrm{~N}$ for the USB drive. In general, applying the proper grasp type to either of the objects demonstrated the effectiveness of offering a variety of grasps, as lower forces and stable grasping could be achieved. The average time required to complete a grasp was approximately 1.5 seconds, depending on the size of the object and grasp type used.

\section{Conclusions}

The combination of EMG classification, high-level grasp planning, and low-level finger control provides an accurate and simple way of controlling a prosthesis' automated grasping motions. With a combination of cylinder, tripod, and lateral grasps, a variety of objects can be picked up and many activities of daily living can be performed. In the two-fingered prosthesis prototype, these grasp types are executed with a single main actuator, due to the inclusion of joint locking mechanisms. Implementation of these control systems and mechanisms would decrease the size and weight of a prosthesis by reducing the number of main actuators, while still allowing for multiple different flexion trajectories for each finger.

\subsection{Future Work}

In order to expand this prototype to a five-fingered, anthropomorphic hand prosthesis, the actuation system will require the most attention. Though the joint locks reduce the number of main actuators needed, a system is needed to distribute the actuator force across all fingers. The development of a thumb opposition system will also be necessary, which may require separate actuation from the main grasping system. A new main actuator should also be implemented, as its current size would be unfit for an anthropomorphic prosthesis. Alternatives to DC motors, such as pneumatic cylinders, should be investigated also.

Patient interaction with the system should be investigated for a proper evaluation of the control system's intuitiveness. This would also allow for real-time EMG data to be evaluated. The current implementation of the low-level controller would be unfit for coordinated grasp control with five fingers; an intrinsically passive controller could be adapted to improve this (Peerdeman et al., 2012b). The joint locking mechanism can still be improved. Specifically, the compliance in the locked joints should be addressed, by ensuring play in the joints and lock mechanisms is minimized. If the joint locks' solenoid actuators can be reduced in size or replaced by a smaller system, this would free up space for larger friction pawls and also reduce the relative effect of play. The control systems and mechanisms demonstrated in this paper have the potential to provide a modern myoelectrically-controlled hand prosthesis with sufficient degrees of freedom to be considered dynamically anthropomorphic, without complicating its control for the user or weighing down the prosthesis with high numbers of actuators. 


\section{References}

Aukes, D., Kim, S., Garcia, P., Edsinger, A., \& Cutkosky, M. (2012). Selectively compliant underactuated hand for mobile manipulation. Proceedings of the 2012 IEEE International Conference on Robotics and Automation (ICRA), 2824-2829. http://dx.doi.org/10.1109/ICRA.2012.6224738

Boere, D., Kallenberg, L., Witteveen, H., Hermens, H., \& Rietman, J. (2010). A multichannel semg method for myoelectric control of a forearm prosthesis. International Society of Electrophysiology and Kinesiology (ISEK). Aalborg, Denmark.

Buchholz, B., \& Armstrong, T. J. (1991). An ellipsoidal representation of human hand anthropometry. Human Factors: The Journal of the Human Factors and Ergonomics Society, 33(4), 429-441.

Carrozza, M., Cappiello, G., Micera, S., Edin, B. B., Beccai, L., \& Cipriani, C. (2006). Design of a cybernetic hand for perception and action. Biological Cybernetics, 95(6), 629-644. http://dx.doi.org/10.1007/s00422-006-0124-2

Chu, J. U., Jung, D. H., \& Lee, Y. J. (2008). Design and control of a multifunction myoelectric hand with new adaptive grasping and self-locking mechanisms. Proceedings of the IEEE International Conference on Robotics and Automation, 743-748. http://dx.doi.org/10.1109/ROBOT.2008.4543294

Hogan, N. (1985). Impedance control: an approach to manipulation. Journal of Dynamic Systems Measurement and Control, 107(1), 1-24. http://dx.doi.org/10.1115/1.3140702

Light, C., \& Chappell, P. (2000). Development of a lightweight and adaptable multiple-axis hand prosthesis. Medical Engineering and Physics, 22(10), 679-684.

Otto Bock. (2011). Michelangelo Hand Technician Product Brochure. January 2011, Web.

Peerdeman, B., Boere, D., Witteveen, H., Huis in 't Veld, R., Hermens, H., Stramigioli, S., ... Misra, S. (2011). Myoelectric forearm prostheses: State of the art from a user requirements perspective. Journal of $\begin{array}{lllll}\text { Rehabilitation Research \& } \quad \text { Development } & \text { (JRRD), } & 48(6), & \text { 719-738. }\end{array}$ http://dx.doi.org/10.1682/JRRD.2010.08.0161

Peerdeman, B., Fabrizi, U., Palli, G., Melchiorri, C., Stramigioli, S., \& Misra, S. (2012). Development of prosthesis grasp control systems on a robotic testbed. Proceedings of the IEEE International Conference on $\begin{array}{lllll}\text { Biomedical Robotics and Biomechatronics (BioRob), } & \text { 1110-1115. }\end{array}$ http://dx.doi.org/10.1109/BioRob.2012.6290289

Peerdeman, B., Pieterse, G. J., Stramigioli, S., Rietman, J. S., Hekman, E. E. G., Brouwer, D. M., \& Misra, S. (2012). Design of joint locks for underactuated fingers. Proceedings of the IEEE International Conference on Biomedical Robotics and Biomechatronics (BioRob), 488-493. http://dx.doi.org/10.1109/BioRob.2012.6290798

Pons, J., Rocon, E., Ceres, R., Reynaerts, D., Saro, B., Levin, S., \& van Moorleghem, W. (2004). The MANUS-HAND dextrous robotics upper limb prosthesis: Mechanical and manipulation aspects. Autonomous Robots, 16(2), 143-163. http://dx.doi.org/10.1023/B:AURO.0000016862.38337.f1

RSLSteeper. (2012). Be Bionic 3 Technical Information. Issue 2, November 2012, Web.

Touch Bionics Inc. (2011). I-limb ultra datasheet. September 2011, Web.

Ulrich, N., Kumar, V., Paul, R., \& Bajcsy, R. (1989). Grasping with mechanical intelligence. Warsaw, Poland: School of Engineering and Applied Science, University of Pennsylvania.

Wettels, N. (2011). Biomimetic Tactile Sensor for Object Identification and Grip Control: A Multi-modal Sensor Mimicking the Human Digit. Saarbrücken, Germany: LAP Lambert Academic Publishing. 